\title{
Structure of the Yeast Mitochondrial Large Ribosomal Subunit
}

\author{
Alexey Amunts, Alan Brown, Xiao-chen Bai, Jose L. Llácer, Tanweer Hussain, Paul Emsley, Fei Long, \\ Garib Murshudov, Sjors H.W. Scheres, V. Ramakrishnan \\ Laboratory of Molecular Biology, Francis Crick Avenue, Cambridge CB2 0QH, UK
}

Mitochondria have specialized ribosomes that diverged greatly from their bacterial and cytoplasmic counterparts. Being responsible for synthesizing mainly highly hydrophobic membrane subunits of the oxidative phosphorylation complexes, mitoribosomes have been evolutionary tuned to specialize in coupling with protein insertion into the membrane and assembly of the respiratory chain. To provide insights into its specialized role and evolutionary development, we solved the structure of yeast mitoribosomal large subunit using cryo-EM [1]. The advent of high-speed direct electron detectors [2,3] and improved algorithms for the classification and alignment of particles [4], allowed us to determine the structure to an overall resolution of $3.2 \AA$ from 47,124 particles, resulting in a nearly complete stereochemically refined atomic model.

The local resolution was better than $3 \AA$ for most of the interior of the large subunit (Fig 1), which enabled a model to be built de novo. The quality of the electron density allowed identification of amino acid sequence directly from the map without any additional information, leading to an unambiguous assignment of 14 specific mitoribosomal proteins to their corresponding densities. For regions with weaker density, a combination of secondary structure prediction, homology modeling and molecular replacement was used. X-ray crystallographic tools were modified for model building, refinement and cross validation of cryo-EM data.

The resulted atomic model for the large subunit reveals locations of 40 proteins and 3000 nucleotides, with overall mass of $1.9 \mathrm{MDa}, 30 \%$ higher than that of the bacterial counterpart (Fig 2). In addition, two of the found proteins were not previously known to be part of mitoribosome. Thus methodologically, this work shows that recent advances in cryo-EM can be used to determine structures of comparable quality to X-ray crystallography but from much smaller amounts of more heterogeneous material.

The structure reveals an extensive network of interactions linking various functional sites, a new exit tunnel, unique elements of the E site and mitoribosome-specific membrane-facing protuberance that might be involved in anchoring the ribosomes to the mitochondrial inner membrane. Such an extensive remodeling of highly conserved features of ribosomes has not been seen in other domains of life and therefore reflects the specialized nature of protein synthesis in mitochondria.

Structurally, most of mitoribosomal proteins are considerably larger, with an average mass of $\sim 25 \mathrm{kDa}$ compared to $\sim 13.5 \mathrm{kDa}$ for their bacterial counterparts. As a result of protein extensions, the number of protein-protein contacts in mitoribosome is not only higher than in bacteria, but also exceeds that observed in the eukaryotic ribosome (Fig 3). Some of the new interactions include bridging different sites of the subunit, whereas others are involved in the architecture of a unique exit tunnel and potentially docking the mitoribosomes to the inner mitochondrial membrane.

One of the most important findings of this work is that yeast mitoribosomes have evolved an altered path of the tunnel through which the nascent protein chain proceeds as it emerges from the ribosome (Fig 4). The tunnel leads to a new exit site located roughly $35 \hat{\mathrm{A}}$ away from where the bacterial tunnel would have emerged. The new exit adjacent to the unique membrane-facing protuberance, suggesting that nascent polypeptide is co-translationally inserted into the mitochondrial inner membrane. This implies that the attachment to a translocon-like entity for must be very different compared to bacterial 
ribosomes. The exit of the tunnel is also wider than in bacteria, possibly to allow co-translational assembly of oxidative phosphorylation complexes.

The entrance to the mitoribosomal tunnel is noticeably narrower than in all previously determined structures of the large subunit. In this part, we identified a structural re-arrangement of rRNA resulting in two newly formed base pairs unique to mitochondria. These pairs bring opposing strands of rRNA closer together by about $8 \AA$ This location is the binding site of macrolide antibiotics, which unlike many aminoglycosides do not bind to mitoribosomes, and thus do not have the same toxicity. A superposition of the macrolide erythromycin in its binding site shows that it would clash with the narrower tunnel entrance in mitochondria, so in mitoribosomes there is a mechanism for resistance to these antibiotics [5].

\section{References:}

[1] A. Amunts et al., Science (in press).

[2] X.-C. Bai, I. S. Fernandez, G. McMullan, S. H. Scheres. eLife 2, (2013), e00461.

[3] X. Li et al,. Nat Methods 10 (2013), p. 584.

[4] S. H. Scheres. J Struct Biol 180, (2012), p. 519.

[5] I acknowledge HFSP for providing the postdoctoral fellowship for this project.
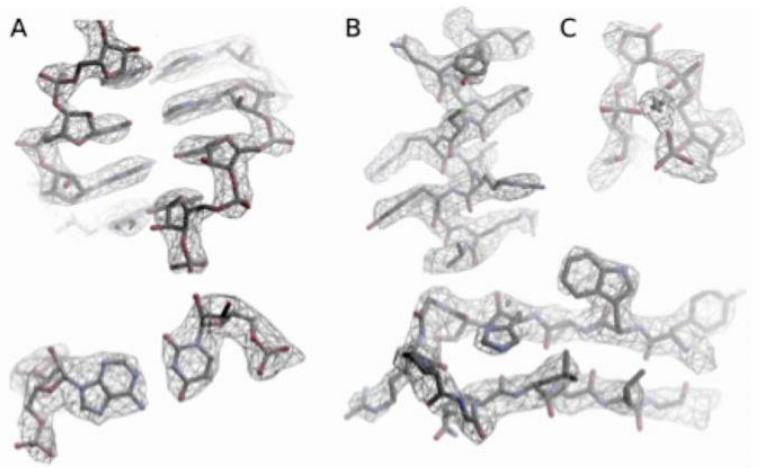

Fig1. Examples of density. A. RNA. B. Proteins. C. Mg ion that coordinates a tight turn in RNA.
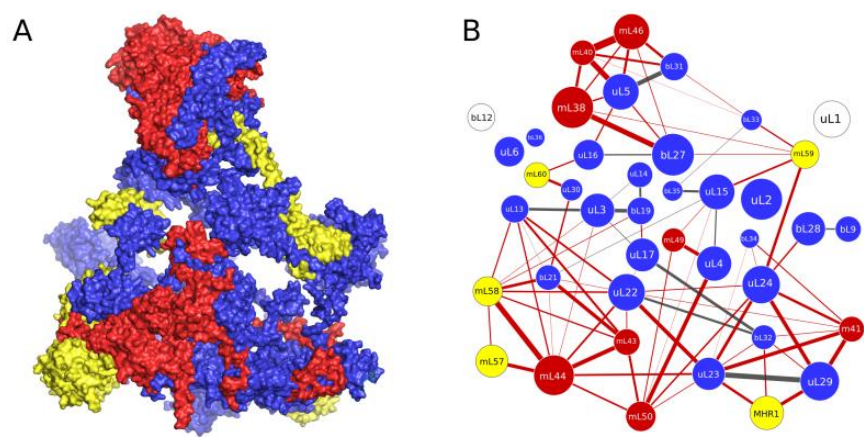

Fig3. Network of interactions of the ribosomal proteins. A. Conserved proteins are in blue, mitochondria specific in red and yellow. B. Schematic representation.

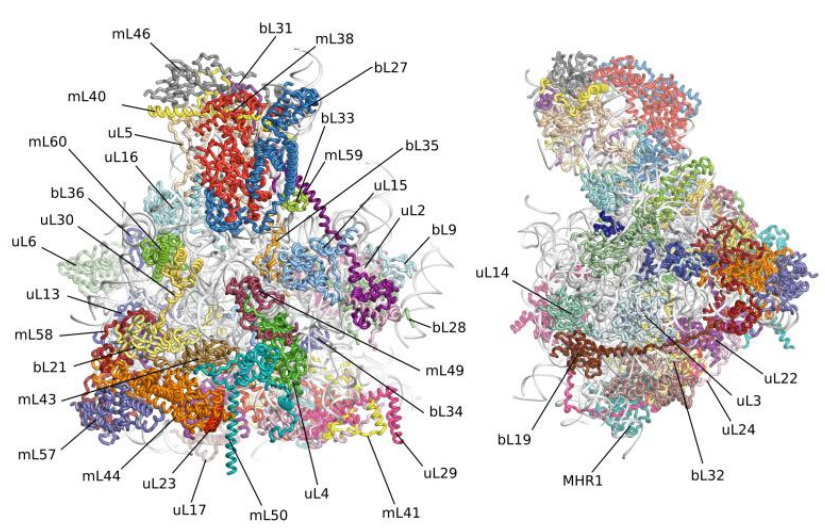

Fig2. Overview of mitoribosomal large subunit. Proteins are coloured.
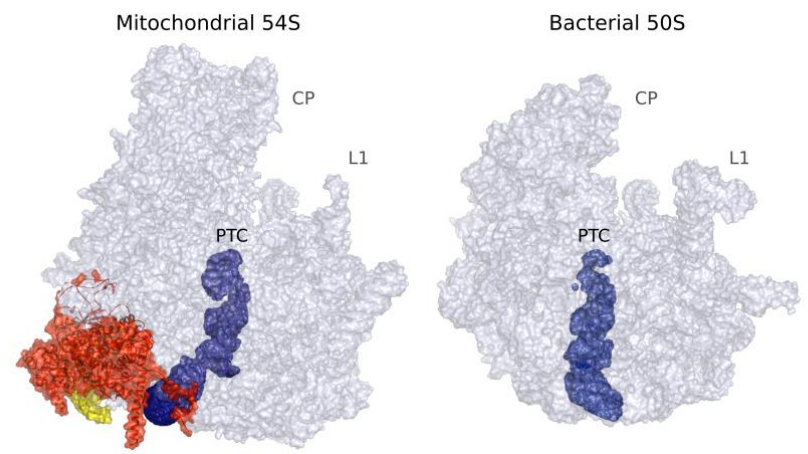

Fig4. Comparison between mitochondrial and bacterial exit tunnel path. The membrane-facing protuberance is shown in red (proteins) and yellow (RNA). 